Annuaire suisse de politique de développement

23-2 | 2004

Les ONG de développement : rôles et perspectives

\title{
Vers une nouvelle politique de la DDC envers les ONG
}

\section{Walter Fust}

\section{(2) OpenEdition}

1 Journals

Édition électronique

URL : http://journals.openedition.org/aspd/460

DOI : 10.4000/aspd.460

ISSN : 1663-9669

Éditeur

Institut de hautes études internationales et du développement

\section{Édition imprimée}

Date de publication : 1 novembre 2004

Pagination : 127-134

ISSN : 1660-5934

\section{Référence électronique}

Walter Fust, «Vers une nouvelle politique de la DDC envers les ONG », Annuaire suisse de politique de développement [En ligne], 23-2 | 2004, mis en ligne le 10 mars 2010, consulté le 08 septembre 2020. URL : http://journals.openedition.org/aspd/460 ; DOI : https://doi.org/10.4000/aspd.460 


\section{Vers une nouvelle politique de la DDC envers les ONG}

L a Confédération met souvent en évidence dans les documents officiels la complémentarité de l'aide des ONG suisses de politique de développement par rapport à l'aide publique au développement, et la signification de la collaboration avec les ONG ainsi que des contributions publiques pour des actions menées par les ONG. Pour compléter différents textes de ce dossier et mieux mettre en évidence les perspectives d'avenir des relations entre Etat et ONG, le comité de rédaction de l'Annuaire a rencontré le directeur de la Direction du développement et de la coopération (DDC), Walter Fust ${ }^{1}$. Dans d'autres articles de ce dossier, nous parlons des formes que revêt le financement public des activités des ONG: mandats spécifiques confiés par la DDC, contributions de programme sur plusieurs années accordées à certaines ONG, financement d'actions humanitaires. La collaboration entre la DDC et les auvres d'entraide remonte à l'origine de l'aide publique dans les années 1950, mais le contexte international a changé ces dernières années et les ONG se trouvent en concurrence croissante avec d'autres acteurs de la coopération comme les entreprises privées, les consultants privés et les $O N G$ des pays du Sud. Une évaluation de la politique de la DDC envers les ONG est en cours en 2004. Cet entretien met en lumière les changements intervenus dans le contexte national et international et leurs implications pour le travail des ONG suisses de développement.

Annuaire: Beaucoup de textes, dont les messages du Conseil fédéral aux Chambres fédérales pour les crédits-cadre de la coopération au développement et de l'aide humanitaire, relèvent l'importance de la société civile et de la collaboration de la DDC avec les ouvres d'entraide. Quelles sont les réflexions de la DDC dans ce domaine pour l'avenir?

Walter Fust: pour la DDC, les ONG sont des partenaires très importantes dans le domaine du développement: elles sont d'une part des interlocutrices appréciées sur le plan de la politique de développement et d'autre part des partenaires de premier plan pour la mise en auvre des programmes de développement. Je parle des ONG dans leur ensemble, des plus petites jusqu'aux plus grandes, des ONG nationales comme des ONG internationales, de celles qui font plutôt du lobbying comme de celles qui se concentrent sur la mise en œuvre de programmes et de projets, de celles qui assurent leur propre fundraising (collecte de fonds) comme de celles qui nous demandent des cofinancements. Je le répète: les ONG sont des interlocutrices et des partenaires importantes pour la DDC.

Actuellement, il y a une nouvelle dimension nationale et régionale dans le monde des ONG, avec aussi une augmentation du nombre des acteurs. Le contexte international dans lequel travaillent les gouvernements change fortement. Aujourd'hui,

1 Interview donnée par Walter Fust, directeur de la DDC, le 3 septembre 2004, en présence de Konrad Specker, Section ONG de la DDC, et de Monika Egger et Gérard Perroulaz, membres du Comité de rédaction de l'Annuaire. 
le discours sur la politique de développement se concentre davantage dans les enceintes spécifiques des Nations unies, dans les institutions de Bretton Woods et dans celles du Comité d'aide au développement (CAD) de l'OCDE. De plus en plus de thèmes qui concernent les programmes spéciaux des Nations unies le PNUD, l'ECOSOC - sont même traités au sein de l'Assemblée générale des Nations unies. Cela implique que les ONG doivent être à la hauteur de ces thèmes et de ce développement.

Les approches de la coopération bilatérale se sont modifiées. On est passé de l'approche par projet à l'approche par programme, puis à l'appui sectoriel et à l'aide budgétaire ${ }^{2}$. Il y a aussi une polarisation accrue. Les Américains et les Japonais, par exemple, ne veulent rien savoir de l'aide budgétaire, mais restent attachés aux dons en forme de programme/projet. Certains ministères ou agences gouvernementales sont passés directement de l'approche par projet à l'approche budgétaire.

La Suisse a choisi un autre chemin. Il y a une douzaine d'années, elle est passée de l'approche par projet à l'approche par programme, qui nécessite un dialogue politique avec nos partenaires respectifs, puis elle a opté partiellement pour des aides budgétaires et l'approche sectorielle. Certains pays comme les Pays-Bas, la Grande-Bretagne ou la Norvège favorisent l'aide budgétaire, de même que l'Union européenne. D'autres pays promeuvent le sector wide approach (approche sectorielle) et ne s'engagent plus dans des projets ou des programmes.

Jusqu'à présent, la Suisse a été considérée par le CAD (OCDE) comme suivant une approche plutôt conservatrice dans le sens que j'ai toujours défendu et que je continuerai à défendre: les différentes approches sont toutes nécessaires et complémentaires - l'approche par projets, l'approche par programmes, l'approche sectorielle et l'approche budgétaire - mais les instruments sont choisis selon les circonstances et selon la concertation entre les divers bailleurs de fonds.

Pour les ONG du Nord, cela implique des changements en matière de mandats. On pourrait réduire toute cette complexité à un constat très simple: en fin de compte, il y aura tout de même des projets sur le terrain. Ces projets sont dirigés par d'autres acteurs que l'agence de développement gouvernementale du Nord et, dans certains cas, par les PVD eux-mêmes. Il y a donc un paradigme qui change pour les ONG du Nord, qui doivent trouver des alliances avec les ONG du Sud pour être des partenaires dans ce nouveau contexte.

Evidemment, les relations entre bailleurs de fonds et ONG ne vont pas changer fondamentalement, mais les ONG doivent se positionner en concertation avec les agences bilatérales et les ministères du développement. Le moment va arriver où l'aide multilatérale prendra plus d'importance, ce qui ne signifie pas forcément

2 L'aide par projet ou par programme est liée à la mise en œuvre concrète d'un projet ou d'un programme (le programme propose une vision stratégique d'ensemble comprenant divers projets). Elle se déroule en premier lieu dans les pays en développement ou en transition, bien que les centrales des institutions bilatérales ou multilatérales ainsi que les ONG concernées puissent y participer dans une large mesure. Selon l'approche sectorielle, les principaux donateurs actifs dans un secteur s'orientent en fonction de la stratégie sectorielle du bénéficiaire (santé, éducation, etc.). L'aide budgétaire est un don alloué à un pays partenaire afin de financer ses dépenses publiques, et notamment les dépenses de lutte contre la pauvreté; elle est dite générale si elle bénéficie à l'ensemble du budget de l'Etat central, et sectorielle lorsque c'est par exemple le Ministère de la santé qui en bénéficie directement. (Sources: sites Internet de la DDC et du seco.) [N.D.E.] 
qu'il y aura moins de possibilités pour les ONG! Mais ce sont des instances différentes qui engageront les ONG: les gouvernements du Sud, les organisations multilatérales...

\section{Annuaire: Faut-il privilégier les contributions aux ONG suisses actives dans la coopération au développement plutôt que celles aux ONG du Sud? Quelle est la politique de la DDC par rapport à un soutien direct à des ONG dans les pays en développement (ou dans les pays en transition), en vue du renforcement de la société civile dans ces pays?}

WF [Les explications suivantes portent sur la question des mandats pour la mise en œuvre des programmes/projets de la DDC]: La tendance à la décentralisation des opérations est un facteur de changement, qui comprend une diminution de la gestion directe par la centrale et une augmentation des compétences et des capacités accordées aux bureaux de coopération (BUCO) de la DDC dans les pays prioritaires de la coopération suisse. Le nombre d'acteurs non gouvernementaux n'augmente pas seulement dans les pays du Nord, mais aussi dans les pays du Sud, où il croît de façon exponentielle. Tôt ou tard chaque acteur va se trouver confronté à la question fondamentale suivante: Quelle est encore sa valeur ajoutée pour contribuer au développement dans les pays concernés? Quelle est la valeur ajoutée de l'ONG du Nord par rapport à l'ONG du Sud?

Les ONG font donc face à des défis, non seulement relativement à des volumes financiers, mais aussi par rapport à la question de la valeur ajoutée qu'elles apportent. Peut-être devraient-elles investir des domaines qui ne sont pas occupés par les ONG du Sud. Ce sujet soulève certainement des divergences d'opinion: une idée largement répandue parmi certaines ONG suisses est que l'Etat devrait mettre davantage de fonds à la disposition des ONG qui collaboreraient ensuite avec les ONG du Sud pour utiliser ces moyens. Je ne suis pas convaincu que les coûts de transfert en seraient a priori réduits, et encore moins que l'influence sur la politique dans les pays bénéficiaires s'en trouverait renforcée.

Quand on travaille avec la société civile, pourquoi passer par une ONG suisse si on peut, à travers un bureau de coopération en Inde ou ailleurs, trouver d'excellentes ONG sur place? Quelle est la valeur ajoutée de l'ONG suisse quant au contenu du travail, sans oublier en outre les coûts additionnels que cela entraîne? Mais il y a des domaines dans lesquels on ne trouve pas de partenaires dans les pays concernés, des domaines très spécifiques, ou des niches, où les ONG suisses s'avèrent nettement plus fortes. Elles doivent ainsi trouver leur position stratégique de succès dans ce contexte; chaque ONG doit se poser cette question, dans un cadre stratégique établi sur cinq à dix ans, tout en tenant compte des vecteurs en mutation.

Nous ne collaborons pas directement avec les ONG du Sud depuis la centrale. Cela passe par nos bureaux de coopération qui choisissent leurs partenaires sur place. C'est une conséquence de la réforme de la DDC et de la décentralisation pour réduire les coûts. Il reste alors à voir de quelle façon les ONG suisses se lient avec le bureau de coopération dans certains pays pour être présentes sur place, ou pour suivre les projets et les programmes dans les secteurs stratégiques qu'on a choisis. C'est la raison pour laquelle nous publions tous nos programmes pluriannuels: il est ainsi facile pour une ONG d'identifier les secteurs 
dans lesquels la DDC s'engage dans les cinq années à venir. Je pense que lors des discussions menées régulièrement par la DDC avec les ONG suisses, nous parvenons aussi à leur montrer quelles sont nos orientations. Je suis tout à fait ouvert aux recommandations des ONG lorsqu'elles aident à améliorer l'impact ou à trouver un bon chemin. Mais je ne suis pas d'accord de maintenir des structures désuètes ou sans avenir.

Les ONG suisses doivent jouer sur leurs avantages comparatifs. Mais je ne suis pas là pour donner mon avis aux $\mathrm{ONG}$, car il s'agit d'un marché. Le monde des ONG n'est pas une sorte de parc pour espèces protégées, comme dans le domaine de la biodiversité. Nous voulons des partenaires forts en Suisse, mais des partenaires qui regardent de l'avant et qui choisissent et développent des capacités dans les domaines où ils ont fait leurs preuves et que l'on ne peut pas trouver sur place au Niger, au Tchad ou ailleurs. On ne peut pas toujours prôner l'importance du renforcement de la société civile dans les pays en développement et à l'Est et ensuite ne pas leur donner les possibilités de se déployer.

Pour en revenir à la question de la décentralisation, oui, une ONG suisse peut être mise dans une situation de concurrence sur les marchés du Sud. Deux cas se présentent. Dans le cas d'un contrat en régie, la concurrence est nette car les ONG suisses doivent montrer qu'elles apportent une réelle valeur ajoutée par rapport aux ONG locales. Si elles y parviennent, notre coordinateur va certainement les choisir. L'autre cas se réfère à des alliances, par exemple entre une ONG du Nord et une ONG du Sud. Ce genre de combinaison va devenir plus fréquent.

Annuaire: Dans le contexte de changement que vous évoquez, pensez-vous que les ONG fassent la même analyse? Comprennent-elles qu'un contexte en mutation implique aussi un changement du comportement des acteurs?

WF: Je pense que certaines ONG l'ont très bien compris. Mais les différentes ONG ont aussi différentes structures de management. Et là il se peut que dans les grandes organisations, avec leurs structures spécifiques (les conseils de fondation par exemple), les changements dans les structures et les approches se fassent plus lentement.

Que veut dire ce changement du contexte en pratique? Il ne signifie nullement qu'il y ait un volume total moins grand pour les ONG suisses. Mais il est clair, par exemple, que la compétition pour l'accès aux fonds qui sont administrés par les Etats du Sud va nettement s'amplifier, et les ONG qui sont bien placées dans un pays où elles travaillent déjà doivent obtenir des «tuyaux» très précis et suivre de près les nouvelles orientations de l'aide.

En bref, l'avenir se montrera plus exigeant pour les ONG. Le monde change et il y a des ONG qui l'ont très bien compris - et d'autres non. Cela peut être un facteur de continuité au sein de certains ONG que de ne pas vouloir changer de stratégie. L'avenir sera nettement plus difficile pour les ONG qui ne savent pas s'orienter ni se positionner dans le contexte international et aussi dans le contexte suisse.

Annuaire: Quels sont selon vous les atouts majeurs des ONG et les principaux arguments qui plaident en faveur d'une collaboration entre la DDC et les ONG, par rapport à une aide publique directe de la Suisse à un gouvernement partenaire du Sud ou de l'Est? 
WF: Je défends pour la DDC «l'approche pour la meilleure solution». Nous ne sommes pas dans la situation où il n'y aurait que l'aide de gouvernement à gouvernement. On reproche à la Suisse d'être la coopération qui travaille le moins directement avec les gouvernements. On nous le reproche. Mais j'assume ces reproches puisque c'est une politique que nous voulons défendre.

Un pilier important de notre collaboration avec les ONG suisses sont les contributions par programme. On nous reproche parfois de cofinancer des programmes d'ONG qui travaillent en dehors de nos pays ou de nos thèmes prioritaires. Mais nous estimons que c'est aux ONG de décider dans quel pays elles veulent intervenir et dans quel secteur elles souhaitent engager des moyens mobilisés par elles-mêmes et complétés par la DDC. La DDC soutient le travail de certaines ONG sur la base des priorités définies par les ONG elles-mêmes, car si les ONG suisses n'avaient plus cette liberté de faire leurs propres choix, elles deviendraient des ONG étatiques, de simples exécutants de projets choisis par nous, des GONGOs (ONG gouvernementales). Les ONG ne doivent pas être de simples exécutants de la politique décidée par la DDC; c'est pourquoi nous tenons à ces contributions de programme.

Le rôle critique des $\mathrm{ONG}$ est précieux pour une démocratie. On nous reproche parfois de financer des activités d'ONG qui par ailleurs peuvent s'exprimer dans des campagnes contre certaines politiques gouvernementales, voire même contre la politique de la DDC. L'indépendance politique des ONG est pour moi importante; nous ne sommes pas là pour aligner et dicter les opinions publiques, nous sommes là pour les soutenir afin qu'elles accomplissent des programmes et des projets intéressants. On ne demande pas non plus de supprimer les subventions ou les paiements directs à des paysans suisses qui seraient opposés aux orientations de la politique agricole suisse ou à la politique de la Suisse défendue à l'OMC. Les ONG peuvent s'exprimer comme elles le veulent, et nous avons notre liberté d'avoir nos opinions lorsque nous sommes convaincus; cela fait partie du débat démocratique où chaque acteur a un rôle à jouer.

Certains nous disent aussi que la DDC se disperse trop, qu'il est trop coûteux de travailler avec autant d'ONG, et souvent de très petite taille. Pour moi, chaque ONG, même petite, peut être importante. L'existence de fédérations cantonales facilite bien entendu notre travail, mais ce n'est pas à nous d'imposer ces regroupements. C'est aux ONG de voir les avantages de ces formules.

A ceux qui nous reprochent de travailler trop étroitement avec les ONG, nous répondons que les ONG nous apportent aussi un savoir que l'on ne pourrait pas forcément développer à l'intérieur de la DDC.

L'ancrage des ONG suisses dans la population suisse est également de grande importance pour la DDC. Elles doivent être portées par la société en Suisse, d'où aussi l'intérêt d'avoir une multitude d'ONG en Suisse et pas seulement quelques grandes organisations.

Je constate que, contrairement à ce que disent certains, «société civile» n'égale pas «ONG». La société civile, c'est les citoyens, la citoyenneté. Si les acteurs non gouvernementaux du développement croient détenir une légitimité du simple fait qu'ils représentent la «société civile», ils se trompent et vont perdre. Beaucoup de gens n'ont pas la rigueur intellectuelle de voir cela clairement. Les ONG représentent une partie seulement de la société civile. Elles sont un acteur parmi 
d'autres, qui représente les intérêts précis d'une partie de la population (par exemple le lobbying pour les intérêts des populations défavorisées). Elles ne représentent pas la société civile telle quelle. Elles ne sont pas élues.

Annuaire: Un autre acteur qui prend de l'importance est le secteur privé, plus habitué à travailler dans un milieu de concurrence. La tendance de la DDC va-t-elle être de favoriser plutôt le privé au détriment des ONG ?

WF: Non. Nous avons ouvert des soumissions pour satisfaire les exigences de l'OMC sur les soumissions publiques. Et ces soumissions sont ouvertes aux compagnies privées. On constate qu'il y a deux types de partenaires de l'économie privée: 1) des entreprises qui participent aux soumissions seulement lorsqu'elles n'ont pas assez de travail sur le marché privé; et 2) des entreprises qui ont une branche souhaitant travailler dans les PVD et y être compétitive par rapport aux ONG.

Annuaire: Quelles sont finalement les différences entre les ONG de développement et les entreprises privées travaillant dans le secteur du développement?

L'entreprise doit assumer le risque si elle n'arrive pas à satisfaire les besoins et à couvrir les coûts, tandis que les ONG calculent les coûts et les facturent à la DDC (frais de gestion). Les coûts d'acquisition sont à peu près les mêmes pour les deux. Certaines compagnies ont peut-être un peu plus de réserves que les ONG, mais il y a aussi des ONG qui ont des réserves. Cependant, relativement peu d'entreprises privées participent à des projets classiques de développement tandis qu'on trouve nettement plus d'instituts universitaires ou d'alliances entre consultants qui créent des consortiums pour gérer des programmes et des projets.

Je ne peux dire que nous menions actuellement une politique qui favorise le secteur privé au détriment des ONG. En définitive, c'est celui qui sait faire le mieux à un prix adéquat, mais avec un résultat et un impact, qu'on choisira. La politique est donc d'ouvrir la compétition pour les nouveaux projets et ce qui a changé, c'est le volume des contrats en régie, qui est en diminution.

Par contre, il faut remarquer que les entreprises privées en Suisse bénéficient beaucoup plus de l'engagement financier de la Suisse envers la Banque mondiale et envers les banques régionales de développement, où elles sont soumises à la compétition internationale. Des entreprises suisses sortent souvent gagnantes de cette compétition. Cet engagement financier de la Suisse a des retombées pour l'économie privée suisse qui vont au-delà de la part suisse du capital de ces institutions financières. Mais il est vrai que rares sont les ONG suisses qui gagnent des contrats de la Banque mondiale ou de l'ONU.

Pour nous résumer, le monde change, le contexte change, il y a plus d'acteurs et plus de compétition. Chacun qui veut être un acteur doit savoir comment il se positionne. La Suisse doit elle aussi se positionner dans ce contexte changeant, notamment dans le système international, pour montrer aux contribuables que l'on engage leur argent de manière effective et avec un impact. 
Annuaire : Nous abordons dans ce dossier la question du contrôle de qualité sur le monde des ONG en Suisse. Faut-il renforcer et étendre le label ZEWO, ou se diriger plutôt vers une certification de type ISO?

WF: Nous sommes reconnaissants pour le travail de la ZEWO, très utile pour nous, mais en même temps nous ne pouvons pas non plus imposer l'obtention du label à des ONG qui vont bien et qui sont bien dirigées. A nos yeux, il est certes important d'avoir ce label, surtout pour les grandes organisations. Mais les toutes petites ONG qui ne peuvent pas se permettre de dépenser 5000 ou 10'000 francs pour payer le label ne doivent pas s'en trouver pénalisées.

(Konrad Specker souligne:) Il y a une tendance à renforcer les certifications et la notation (rating): SGS, ISO et autres. Certaines ONG privilégient ce type de certification. Je pense que la certification est une bonne chose mais qu'elle ne représente qu'un seul élément de la gestion de la qualité. L'expérience montre qu'une organisation qui bénéficie par exemple d'un «triple A» de la SGS n'est pas forcément la meilleure au niveau de la pertinence de son action, ni la meilleure des organisations. Tout partenariat nécessite aussi et surtout un dialogue. Une organisation qui est capable d'analyser ses problèmes et d'avouer ses propres faiblesses, de mettre ces aspects sur la table et d'en discuter, fait un meilleur travail qu'une ONG qui se proclame parfaite sur la base d'une certification ou d'une notation.

Annuaire: L'évaluation externe en cours sur la politique de la DDC envers les ONG suscite des inquiétudes au sein du monde des ONG, en raison par exemple des menaces de coupures importantes dans les contributions de la DDC aux ONG. Que pouvez-vous dire sur cette évaluation en cours?

WF: L'évaluation qui a été menée en été 2004 est une évaluation de la politique de la DDC envers les ONG, et non pas, comme certains le craignent, une évaluation du travail des ONG. Il n'était absolument pas question pour nous d'évaluer le travail des ONG, mais d'examiner la politique de la DDC, ou plutôt le problème, à l'intérieur de la DDC, des différentes politiques de collaboration avec les ONG, car nous avons dû constater qu'il y a des politiques différentes dans les domaines de la coopération avec le Sud, de la coopération avec les pays de l'Est et de l'action humanitaire. N'y aurait-il pas une politique à définir et à harmoniser? Le rapport d'évaluation est discuté au sein de la DDC et avec les principales ONG avant d'arriver à une conclusion finale. En décembre 2004, la DDC discutera en interne de cette évaluation et celle-ci devrait nous aider à mieux définir une politique cohérente de la DDC envers les ONG.

\section{Annuaire: Pourquoi cette évaluation maintenant?}

WF: Parce que, comme nous l'avons vu, le contexte international de la coopération a changé. La DDC doit procéder à des choix quant à ses approches: doitelle continuer à pratiquer une approche combinée entre instruments par projet/programme, aide sectorielle et aide budgétaire, ou doit-elle se concentrer sur l'aide sectorielle? Faut-il favoriser le bilatéral ou le multilatéral? 
Annuaire: Mais comprenez-vous que certaines ONG puissent avoir des craintes quant à l'avenir des relations entre la DDC et les ouvres d'entraide?

WF: Je le répète encore une fois: la société civile en tant qu'acteur du développement est un partenaire extrêmement important, mais le fait d'être partenaire dans l'exécution de projets ne donne pas une garantie de l'être aussi dans dix, quinze ou vingt ans. Les ONG aussi doivent changer, comme nous devons changer. Nous trouverons toujours des personnes qui s'opposent au changement ou à l'adaptation, dans chaque entreprise, dans chaque ONG, et aussi à la DDC.

\section{Annuaire : Pour terminer, avez-vous un message à lancer aux ONG suisses?}

WF: Regardez vers le futur! Croyez en vous-mêmes! Soyez innovatrices! J'ai créé le slogan Il faut contredire le pessimisme théorique par un optimisme pragmatique. Il faut regarder en avant; connaître le passé, connaître le présent, mais regarder en avant. Il faut se positionner. Ce n'est pas un bon principe de regarder tout le temps dans le rétroviseur. Il faut regarder en avant pour trouver le chemin, établir des alliances et être fort. 